\title{
BMJ Open The relationship between the number of cardiologists and clinical practice patterns in acute heart failure: a cross-sectional observational study
}

Noriko Sasaki, ${ }^{1}$ Susumu Kunisawa, ${ }^{1}$ Tetsuya Otsubo, ${ }^{1}$ Hiroshi Ikai, ${ }^{1}$
Kiyohide Fushimi, ${ }^{2}$ Yoshio Yasumura, ${ }^{3}$ Takeshi Kimura, ${ }^{4}$ Yuichi Imanaka ${ }^{1}$

To cite: Sasaki N, Kunisawa S, Otsubo T, et al. The relationship between the number of cardiologists and clinical practice patterns in acute heart failure:

a cross-sectional

observational study. BMJ Open 2014:4:e005988. doi:10.1136/bmjopen-2014005988

- Prepublication history and additional material is available. To view please visit the journal (http://dx.doi.org/ 10.1136/bmjopen-2014005988)

Received 27 June 2014 Revised 2 October 2014 Accepted 20 October 2014

CrossMark

For numbered affiliations see end of article.

Correspondence to Dr Yuichi Imanaka; imanaka-y@umin.net

\section{ABSTRACT}

Objectives: Despite the increasing burden of acute heart failure (AHF) on healthcare systems, the association between centralised cardiovascular specialist care and the quality of AHF care remains unknown. We examine the relationship between the number of cardiologists per hospital and hospital practice variations.

Design, setting and participants: In a retrospective observational study, we analysed 38668 patients with AHF admitted to 546 Japanese acute care hospitals between 2010 and 2011 using the Diagnosis Procedure Combination administrative claims database. Sample hospitals were categorised into four groups according to the number of cardiologists per facility (none, 1-4, 5-9 and $\geq 10$ ). To confirm the capability of administrative data to identify patients with AHF, the $\geq 10$ cardiologists group was compared with two recent clinical registries in Japan. Main outcome measures: Using multivariable logistic regression models, patient risk-adjusted in-hospital mortality rates and age-sex-adjusted ORs of various AHF therapies were calculated and compared among four hospital groups.

Results: The $\geq 10$ cardiologists group of hospitals from the administrative database had similar major underlying disease incidence and therapeutic practices to those of the clinical registry hospitals. Age-adjusted and sex-adjusted ORs of various AHF therapies in the four hospital groups revealed wide practice variations associated with the number of cardiologists. Adjusted in-hospital mortality demonstrated a negative association with the number of cardiologists. In addition, the different hospital-level distribution patterns of specific therapeutic practices illustrated the diffusion process of therapies across facilities.

Conclusions: Wide practice variations in AHF care were associated with the number of cardiologists per facility, indicating a possible relationship between the quality of AHF care and manpower resources. The provision of recommended therapies increased together with the number of cardiologists.

\section{INTRODUCTION}

The high morbidity, mortality and readmission rates in patients with acute heart failure

\section{Strengths and limitations of this study}

- This study uses a large administrative database to provide novel insight into the practice variations in acute heart failure (AHF) care across Japanese hospitals categorised by the number of cardiologists.

- These findings can support improvements to hospital quality of care for patients with AHF from the perspective of health policy.

- Generalisability of conclusions outside of Japan may be limited due to different clinical circumstances across countries.

(AHF) place a heavy burden on healthcare systems, especially in developed countries with ageing populations. ${ }^{1}{ }^{2}$ The association between centralised cardiovascular specialist care and the quality of AHF care remains unknown. Also, the relation between hospital practice variations and the number of cardiologists is still unclear.

Currently, there are only a few clinical registries that have contributed descriptive analyses of AHF cases in Japan ${ }^{3-6}$ including the Acute Decompensated Heart Failure Syndromes (ATTEND) registry $^{3}{ }^{4}$ and the Japanese Cardiac Registry of Heart Failure in Cardiology (JCARE-CARD) ${ }^{5}{ }^{6}$ However, the hospitals included in these registries are likely to be biased towards bigger hospitals with a larger number of cardiologists, which may not be representative of all patients with AHF. Little information exists concerning the hospital management of AHF, based on analyses that encompass wide regions across Japan.

Recently, a code designating 'acute exacerbation' of heart failure (HF), which was newly added in 2009 and unique to the Japanese Diagnosis Procedure Combination (DPC) patient case-mix classification system, ${ }^{78}$ has 
enabled researchers to distinguish AHF from chronic HF. Yet the reliability of these extracted data for clinical or epidemiological analyses remains unclear because of the complexity of AHF itself. ${ }^{2}$

The objective of our study consisted of two steps. First, we examined whether demographics of patients with AHF identified by administrative data using the new code are comparable with those from the aforementioned Japanese registries. These registries were deemed suitable for cross-reference because they were based on clinical data and their data collection period corresponded with that of our study. Second, in order to elucidate the relationship between cardiologists and quality of care, we investigated patient with AHF characteristics, therapeutic process of care, patient outcomes and therapeutic practice patterns among hospital groups stratified by the number of cardiologists per facility.

\section{METHODS}

\section{Data sources}

Data for analysis were extracted from the DPC administrative database, ${ }^{78}$ which contains inpatient information such as patient case-mix, processes of care, medical charges and patient outcomes including mortality. In the DPC system, the code designating 'acute exacerbation' of HF and the determination of the New York Heart Association (NYHA) functional class at admission are determined only by attending physicians and not by other medical or administrative staff; this may provide face validity for the accuracy of these codes. Subsequently, the results of our sample using administrative data and the results of the ATTEND and the JCARE-CARD registries were compared.

The ATTEND registry included patients with AHF from 2007 to 2011. This registry contained 4842 patients from 53 hospitals; patients who met the modified Framingham criteria ${ }^{9}$ were included, but those who had acute coronary syndromes were excluded. ${ }^{3}{ }^{4}$ A preliminary report based on 1110 patients from 32 hospitals of the registry had been previously published, ${ }^{3}$ and we utilised the results of both reports because we observed statistically significant differences in patient characteristics between the two.

The JCARE-CARD registry included patients hospitalised with worsening HF, identified using Framingham criteria. This study enrolled 2675 patients from 164 hospitals between 2004 and 2005, ${ }^{5}{ }^{6}$ and analysed patients with reduced and preserved ejection fraction (EF) ${ }^{6}$ The number of cardiologists per hospital was obtained from the Japanese Circulation Society (JCS) website, ${ }^{10}$ which gives a detailed information on JCS-certified cardiologists.

\section{Study population}

Using the DPC administrative database, we identified a total of 57353 AHF cases who had been admitted to 912 hospitals between 1 July 2010 and 31 March 2011. The selection criteria were (1) a primary diagnosis of $\mathrm{HF}$ (ICD-10 code I50.x), (2) a DPC system code designating an 'acute exacerbation' of HF, (3) NYHA functional class II or higher, and (4) older than 20 years of age. The exclusion criteria are described in online supplementary figure S1. Data at patient level were collected in relation to the context, use and coding of administrative data. Exclusion criteria for hospitals were also used, because these hospitals were assumed to provide less emergency care and thought to be unsuitable for comparisons with hospitals providing high-quality emergency care. As more than two-thirds of all 8565 hospitals in Japan have fewer than 200 beds, ${ }^{11}$ we took these factors into consideration in order to make valid comparisons. The final sample size comprised 38668 patients from 546 hospitals, ranging from 20 patients to 343 patients per hospital.

To perform valid comparisons between the sample hospitals with the clinical registries, our study sample was divided into four groups according to the number of registered cardiologists per hospital (no cardiologist; 1-4; $5-9$; and $\geq 10$ cardiologists); the $\geq 10$ cardiologists group was compared with the registries, as hospitals in both these groups were likely to be similar in both hospital and patient characteristics, as well as medical practice patterns. Subsequently, patient characteristics, outcomes and therapeutic interventions among the four groups in our study sample were examined. To investigate the relationship between cardiologist numbers and quality of hospital care, the age-adjusted and sex-adjusted ORs of specific clinical practices were calculated for each group, using the 1-4 cardiologists group as the reference.

\section{Statistical analysis}

Means and SDs were calculated for continuous data, whereas categorical data were expressed as percentages. Comparisons between the $\geq 10$ cardiologists group in our study sample and the registry groups were performed using the $\chi^{2}$ tests for dichotomous variables.

The age-adjusted and sex-adjusted ORs and 95\% CIs of specific clinical practices among the hospital groups stratified by the number of cardiologists per hospital were analysed using multivariable logistic regression analyses. The risk-adjusted mortality rate was calculated as the ratio of observed mortality to predicted mortality, multiplied by the overall mean mortality rate of $7 \%$. The predicted mortality of each patient was obtained using the predictive model that we had previously reported. Independent variables in this model included 11 patient factors such as age, NYHA functional class and comorbidities. Two-tailed $\mathrm{p}$ values below 0.05 were considered statistically significant. Statistical computations were performed using SPSS software, V.19.0J (SPSS Inc, Chicago, Illinois, USA).

\section{RESULTS}

The baseline characteristics of the hospitals and patients with AHF from the two clinical registries and from the 
study sample based on the administrative database are described in table $1 .^{3-6}$ Our study sample consisted of hospitals from all 47 prefectures in Japan, varying in hospital bed size, case volume, teaching status and ownership (public/private).

At the overall patient level, the mean age and the proportion of male patients in our sample were 78 years and $51 \%$, respectively. Ischaemic heart disease (IHD) was present in approximately $31 \%$, similar to the registries. The observed in-hospital mortality rate was $7 \%$, which was within the range reported in several recent AHF registries. ${ }^{3} 41213$ The median length of stay was similar to the ATTEND registry (18 and 21 days).

\section{Comparisons of patient characteristics and therapeutic practices between the administrative database and the two clinical registries}

The median number of cardiologists and hospital beds, and the proportion of university hospitals in the $\geq 10$ cardiologists group in our study sample were similar to those of the ATTEND registry (table 1).

Details of therapeutic practices as process-of-care measures for hospitalised patients with AHF are shown in table 2. Data for these therapies were not available from the JCARE-CARD registry. Although many differences were statistically significant because of the large sample sizes, the proportions of non-pharmacological interventions and intravenous medications were similar between the $\geq 10$ cardiologists group and the ATTEND registry in many respects. However, the frequencies of percutaneous coronary intervention (PCI), coronary artery bypass grafting (CABG), intra-aortic balloon pump (IABP) and pacemaker implantation (PMI) were much lower in our sample. The proportion of discharge medications was similar to that of the registries.

\section{Comparisons among the four hospital groups from the administrative database stratified by the number of cardiologists}

AHF case volume per hospital, the proportions of male patients, and underlying IHD were observed to decline together with the number of cardiologists (table 1). In contrast, case volume per cardiologist increased with decreasing cardiologist numbers. The $\geq 10$ cardiologists group showed the highest proportion of university hospitals and patients with NYHA class II at admission among the four groups.

With regard to outcome measures, crude in-hospital mortality tended to increase in hospitals with fewer cardiologists, from $4.4 \%$ in the $\geq 10$ cardiologists group to $16.4 \%$ in the group with no cardiologists. Even after adjusting for patient severity factors mentioned in our previous study, ${ }^{7}$ higher likelihood of mortality was still observed in hospitals with fewer cardiologists, from 5.4\% in the $\geq 10$ cardiologists group to $10.7 \%$ in the group with no cardiologists (figure 1).

All non-pharmacological interventions during hospitalisation showed reductions in relation to decreasing numbers of cardiologists. Also, major intravenous and discharge medications also tended to decline with decreasing numbers of cardiologists (table 2).

When examining the effects of cardiologist numbers in processes of care such as therapeutic interventions, there were wide practice variations at the cardiologiststratified hospital group level, as shown by the age-adjusted and sex-adjusted ORs (table 3). The group of hospitals with no cardiologists tended to show lower ORs for each therapeutic intervention. In contrast, groups with 5-9 and $\geq 10$ cardiologists had generally higher ORs, especially in specific interventions or medications used to treat severe patients such as intubation, right-heart catheterisation, cardiac resynchronisation therapy, implantable cardioverter-defibrillator, IABP and intravenous carperitide use. Conventional care such as intravenous dopamine, intravenous digoxin, and digitalis at discharge were lower in the $\geq 10$ cardiologists group, whereas nitrates and digitalis at discharge were higher in the group with no cardiologists.

In addition, wide therapeutic practice variations at the individual hospital level were observed among and within the four hospital groups. We found three distinct hospital distribution patterns for specific therapeutic interventions (figure 2). These patterns were as follows; (type A) a convex inclination pattern representing commonly used therapies for AHF such as intravenous diuretics, ACE inhibitors/angiotensin-receptor blockers and warfarins; (type B) a linear inclination or a combination pattern of the other two types representing an intermediate distribution stage of specific therapy use such as intravenous carperitide, heparin and $\beta$-blockers at discharge; and (type C) a concave inclination pattern representing less commonly used therapies such as intravenous dobutamine, intubation, PCI and oral inotropic agents.

\section{DISCUSSION}

In this study, we confirmed the compatibility of administrative data to properly identify hospitalised patients with AHF by cross-referencing the results from recent clinical registries, and further revealed wide practice variations in AHF care among hospitals in association with the number of cardiologists per facility.

Major underlying diseases, major therapeutic interventions and proportions of discharge medications showed approximate similarities between the $\geq 10$ cardiologists group and the clinical registries. These general similarities indicate that our study sample is comparable with the cohorts from the clinical registries. The results were consistent with a prior study that compared CABG cases between administrative data and registry data, which demonstrated that major comorbidities were similarly prevalent between the two data sets. ${ }^{14}$ Since several disparities were also detected among the three cohorts of the clinical registries, the differences between our sample and the clinical registries appeared to be 
Table 1 Baseline characteristics of hospitals and patients with AHF

\begin{tabular}{|c|c|c|c|c|c|c|c|c|}
\hline \multirow[b]{3}{*}{ Characteristics } & \multicolumn{3}{|l|}{ Clinical registries } & \multicolumn{5}{|c|}{ Study sample (administrative database) } \\
\hline & \multirow{2}{*}{$\begin{array}{l}\text { ATTEND } \\
\text { Preliminary report }^{3}\end{array}$} & \multirow[b]{2}{*}{ ATTEND ${ }^{4}$} & \multirow[b]{2}{*}{ JCARE-CARD $^{56}$} & \multicolumn{5}{|c|}{ Hospital subgroups stratified by the number of cardiologists per facility } \\
\hline & & & & $\geq 10$ & $5-9$ & $1-4$ & 0 & Overall \\
\hline Geographic region (number of prefectures) & 20 & 24 & 47 & 27 & 45 & 45 & 22 & 47 \\
\hline \multicolumn{9}{|l|}{ Institutional level } \\
\hline Number of hospitals & 32 & 52 & 164 & 72 & 185 & 263 & 26 & 546 \\
\hline Hospital beds, mean (SD) & $557(337)$ & 564 (332) & NA & $712(264)$ & $523(224)$ & $364(154)$ & $204(76)$ & $456(234)$ \\
\hline University hospitals, \% & 41 & 40.4 & NA & 63.9 & 13.0 & 4.9 & 0 & 15.6 \\
\hline Certified*training facilities, $\%$ & 93.8 & 90.4 & 100 & 100.0 & 100.0 & 74.5 & 0.0 & 91.9 \\
\hline Number of cardiologists*/facility, median & 9.5 & 9 & NA & 13 & 6 & 3 & 0 & 4 \\
\hline Total patients & 1110 & 4842 & 2675 & 6509 & 15337 & 15867 & 955 & 38668 \\
\hline Case volume/year & - & - & - & 8679 & 20449 & 21556 & 1273 & 51557 \\
\hline Case volume/facility·year, mean(SD) & - & - & - & $120.5(82.6)$ & $110.5(52.1)$ & $80.4(41.6)$ & $49.0(21.1)$ & $94.4(55.0)$ \\
\hline Case volume/facility.year.cardiologist, mean(SD) & - & - & - & $9.0(6.5)$ & $17.2(7.9)$ & 34.4 (22.9) & - & $24.8(19.9)$ \\
\hline \multicolumn{9}{|l|}{ Patient level } \\
\hline Age, mean years (SD) & $73(14)$ & $73(14)$ & $71(13)$ & $75.3(12.9)$ & $77.2(12.1)$ & $78.9(11.6)$ & $81.3(10.7)$ & $77.7(12.1)$ \\
\hline Male, \% & 59 & 58.0 & 60 & 57.2 & 51.7 & 49.1 & 44.0 & 51.4 \\
\hline NYHA functional class at admission, $\%$ & $n=1092 \dagger$ & $n=4699 \dagger$ & $n=2644 \dagger$ & & & & & \\
\hline II & 12.3 & 16.1 & 11.5 & 33.8 & 29.0 & 25.6 & 22.8 & 28.3 \\
\hline III & 39.7 & 38.9 & 45.1 & 38.9 & 37.6 & 39.2 & 35.4 & 38.4 \\
\hline IV & 48.0 & 45.0 & 43.4 & 27.3 & 33.4 & 35.2 & 41.8 & 33.3 \\
\hline Underlying diseases, \% & & & $n=1692$ & & & & & \\
\hline Ischaemic heart disease & $33 \ddagger$ & $31.1 \ddagger$ & 32.0 & 34.6 & 31.0 & 30.3 & 21.9 & 31.1 \\
\hline Atrial fibrillation/flutter & 40 & 39.6 & 35.0 & 26.3 & 27.3 & 28.2 & 22.7 & 27.4 \\
\hline Cardiomyopathy & NA & 12.7 & 26.2 & 8.8 & 7.1 & 5.5 & 2.5 & 6.6 \\
\hline Valvular heart disease & NA & 19.4 & NA & 16.7 & 16.3 & 15.4 & 9.1 & 15.8 \\
\hline Hypertension & 71 & 69.4 & 52.6 & 53.6 & 55.9 & 54.8 & 37.8 & 54.6 \\
\hline Diabetes mellitus & 34 & 33.8 & 29.8 & 24.8 & 24.3 & 26.2 & 19.3 & 25.0 \\
\hline Previous history of stroke & 12 & 14.0 & 14.7 & 4.3 & 5.3 & 7.1 & 7.7 & 5.9 \\
\hline Renal failure (mild to moderate) & NA & NA & 11.7 & 9.6 & 10.4 & 10.8 & 10.2 & 10.4 \\
\hline COPD & 9 & 9.5 & 6.5 & 5.3 & 6.6 & 6.9 & 5.0 & 6.5 \\
\hline \multicolumn{9}{|l|}{ Outcomes } \\
\hline Mean (median) length of stay, days & $31(21)$ & $30(21)$ & $35.6(\mathrm{NA}) / 31.2(\mathrm{NA}) \S$ & $21.7(18.0)$ & $21.7(17.0)$ & $22.2(18.0)$ & $22.9(17.0)$ & $21.9(18.0)$ \\
\hline Crude in-hospital mortality, \% & 7.7 & 6.4 & $3.9 / 6.5 \S$ & 4.4 & 6.8 & 7.6 & 16.4 & 7.0 \\
\hline
\end{tabular}

${ }^{*}$ Certified by the Japanese Circulation Society.

†The number was recalculated by subtracting the original NYHA class I patients.

FWithout acute coronary syndromes. ${ }^{3} 4$

§Length of hospital stay with reduced left ventricular ejection fraction $(\mathrm{EF}) /$ preserved $\mathrm{EF}^{6}$

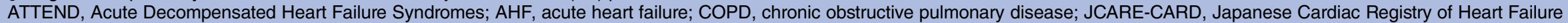

in Cardiology; NA, not available; NYHA, New York Heart Association. 
Table 2 Clinical practices in patients with AHF

Clinical registries

Study sample (administrative database)

Hospital subgroups stratified by the number of

cardiologists per facility

\begin{tabular}{|c|c|c|c|c|c|c|c|c|}
\hline Therapeutic interventions (\%) & $\begin{array}{l}\text { ATTEND preliminary report } \\
\mathrm{n}=1110\end{array}$ & $\begin{array}{l}\text { ATTEND } \\
\mathrm{n}=4842 \\
\end{array}$ & $\begin{array}{l}\text { JCARE-CARD }{ }^{5} 6 \\
\mathrm{n}=1613\end{array}$ & $\begin{array}{l}\geq 10 \\
n=6509\end{array}$ & $\begin{array}{l}5-9 \\
n=15337\end{array}$ & $\begin{array}{l}1-4 \\
n=15867\end{array}$ & $\begin{array}{l}0 \\
\mathrm{n}=955\end{array}$ & $\begin{array}{l}\text { Overall } \\
n=38668\end{array}$ \\
\hline \multicolumn{2}{|l|}{ Non-pharmacological interventions } & \multicolumn{7}{|l|}{$(n=4842)$} \\
\hline Intubation & 11.1 & 7.5 & - & 12.2 & 9.9 & 8.4 & 6.1 & 9.6 \\
\hline Right heart catheterisation & 20.1 & 16.7 & - & 17.6 & 12.9 & 9.0 & 2.2 & 11.7 \\
\hline Percutaneous coronary intervention & 9.6 & 8.0 & - & 4.3 & 3.4 & 3.2 & 0.4 & 3.4 \\
\hline Coronary artery bypass grafting & 1.4 & 1.3 & - & 0.3 & 0.1 & 0.1 & - & 0.1 \\
\hline Pacemaker & 4.7 & 3.8 & - & 1.5 & 1.0 & 1.1 & 0.6 & 1.1 \\
\hline CRT or CRT-D & 2.4 & 2.3 & - & 1.7 & 0.7 & 0.2 & - & 0.6 \\
\hline Implantable cardioverter-defibrillator & 2.6 & 2.6 & - & 0.3 & 0.1 & 0.1 & - & 0.1 \\
\hline Intra-aortic balloon pump & 3.6 & 2.5 & - & 0.9 & 0.7 & 0.4 & 0.1 & 0.6 \\
\hline Percutaneous cardiopulmonary support & 0.6 & 0.7 & - & 0.4 & 0.3 & 0.1 & - & 0.2 \\
\hline \multicolumn{2}{|l|}{ Intravenous medications } & \multicolumn{7}{|l|}{$(n=4842)$} \\
\hline Diuretics & 80.4 & 76.3 & - & 72.3 & 76.4 & 75.6 & 70.9 & 75.2 \\
\hline Carperitide & 69.4 & 58.2 & - & 59.0 & 49.3 & 41.0 & 19.1 & 46.8 \\
\hline Heparin & NA & NA & - & 60.1 & 54.7 & 44.8 & 25.7 & 50.8 \\
\hline ISDN & 9.2 & 14.5 & - & 25.8 & 21.2 & 18.2 & 8.3 & 20.4 \\
\hline NTG & 26.0 & 20.8 & - & 16.9 & 16.3 & 12.4 & 9.1 & 14.6 \\
\hline ISDN or NTG & NA & NA & - & 36.8 & 32.6 & 27.6 & 15.9 & 30.8 \\
\hline Nicorandil & 10.6 & 9.6 & - & 6.4 & 5.2 & 4.3 & 0.8 & 4.9 \\
\hline \multicolumn{9}{|l|}{ Inotropes } \\
\hline Dobutamine & 12.7 & 11.3 & - & 13.1 & 12.7 & 8.8 & 6.0 & 11.0 \\
\hline Dopamine & 11.0 & 8.8 & - & 9.9 & 14.3 & 13.4 & 10.9 & 13.1 \\
\hline Norepinephrine & 6.2 & 4.7 & - & 6.8 & 5.9 & 4.7 & 4.8 & 5.5 \\
\hline Milrinone & 2.8 & 3.3 & - & 2.3 & 2.3 & 2.4 & 0.8 & 2.3 \\
\hline Olprinone & 0.7 & 0.8 & - & 1.5 & 0.6 & 0.7 & 0.3 & 0.8 \\
\hline Digoxin & 6.5 & 6.9 & - & 6.6 & 8.1 & 7.6 & 7.6 & 7.7 \\
\hline Calcium channel blockers & 8.2 & NA & - & 8.5 & 5.4 & 3.9 & 2.6 & 5.2 \\
\hline \multicolumn{2}{|l|}{ Discharge medications } & \multicolumn{7}{|l|}{$(n=4530)$} \\
\hline Diuretics & 84.5 & 82.3 & 87.0 & 72.0 & 72.2 & 69.3 & 63.7 & 70.8 \\
\hline ACEls & 26.3 & 30.6 & 38.7 & 23.3 & 19.2 & 18.7 & 8.8 & 19.4 \\
\hline $\mathrm{ARBs}$ & 54.5 & 46.0 & 46.4 & 35.2 & 33.9 & 31.0 & 24.6 & 32.7 \\
\hline ACEIs or ARBs & 78.0 & 74.7 & 79.1 & 57.1 & 51.6 & 48.1 & 32.9 & 50.6 \\
\hline Aldosterone receptor blockers & 49.0 & $43.0^{*}$ & $42.2^{*}$ & 42.6 & 38.7 & 34.9 & 24.6 & 37.4 \\
\hline Digitalis & 27.2 & 14.7 & 27.2 & 11.2 & 13.2 & 12.6 & 14.6 & 12.7 \\
\hline$\beta$-blockers & 63.6 & 67.4 & 57.5 & 52.3 & 43.7 & 36.9 & 20.9 & 41.8 \\
\hline Nitrates & 25.5 & 22.4 & 23.0 & 14.4 & 14.4 & 15.1 & 20.0 & 14.8 \\
\hline Calcium channel blockers & 29.1 & 26.8 & 25.4 & 23.0 & 23.3 & 21.1 & 19.9 & 22.3 \\
\hline Statins & 37.3 & 35.6 & 21.0 & 26.9 & 23.6 & 19.4 & 10.4 & 22.1 \\
\hline
\end{tabular}



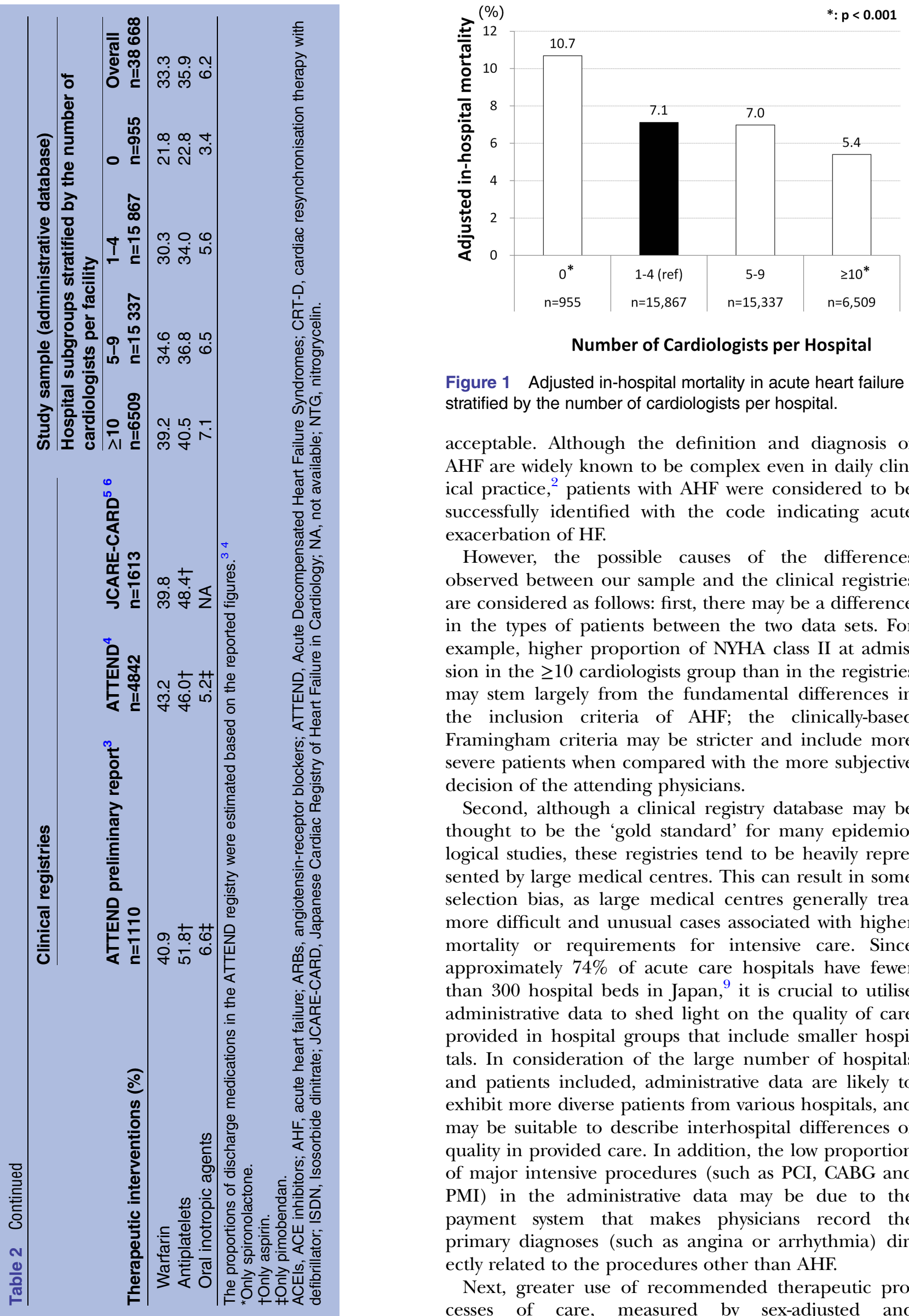
Table 3 Adjusted ORs (95\% Cls) of clinical practices in patients with AHF

\begin{tabular}{|c|c|c|c|c|}
\hline \multirow[b]{3}{*}{ Variables (\%) } & \multicolumn{4}{|c|}{ Study sample (administrative database) } \\
\hline & \multicolumn{4}{|c|}{ Hospital subgroups by the number of cardiologists } \\
\hline & $\begin{array}{l}\geq 10 \\
n=6509\end{array}$ & $\begin{array}{l}5-9 \\
n=15337\end{array}$ & $\begin{array}{l}1-4 \\
n=15867\end{array}$ & $\begin{array}{l}0 \\
n=955\end{array}$ \\
\hline \multicolumn{5}{|l|}{ In-hospital managements } \\
\hline \multicolumn{5}{|l|}{ Non-pharmacological interventions } \\
\hline Intubation & $1.43(1.30$ to 1.57$)$ & $1.16(1.07$ to 1.25$)$ & ref & 0.74 (0.56 to 0.97$)$ \\
\hline Right heart catheterisation & 1.84 (1.69 to 2.01$)$ & $1.34(1.25$ to 1.45$)$ & ref & $0.26(0.17$ to 0.40$)$ \\
\hline Percutaneous coronary intervention & 1.23 (1.06 to 1.43$)$ & $1.02(0.90$ to 1.16$)$ & ref & 0.14 (0.05 to 0.38$)$ \\
\hline Pacemaker & $1.47(1.15$ to 1.89$)$ & $0.94(0.76$ to 1.17$)$ & ref & $0.55(0.24$ to 1.24$)$ \\
\hline ICD & 5.19 (2.31 to 11.69$)$ & $2.48(1.10$ to 5.57$)$ & ref & - \\
\hline CRT or CRT-D & 8.98 (5.81 to 13.89$)$ & 4.08 (2.64 to 6.31$)$ & ref & - \\
\hline Coronary artery bypass grafting & 4.95 (2.28 to 10.79$)$ & $1.98(0.89$ to 4.37$)$ & ref & - \\
\hline Intra-aortic balloon pump & $1.96(1.36$ to 2.82$)$ & 1.57 (1.14 to 2.17$)$ & ref & $0.33(0.05$ to 2.36$)$ \\
\hline Percutaneous cardiopulmonary support & 2.47 (1.41 to 4.31$)$ & 1.62 (0.97 to 2.72$)$ & ref & - \\
\hline \multicolumn{5}{|l|}{ Intravenous drugs } \\
\hline Diuretics & 0.87 (0.82 to 0.93$)$ & 1.06 (1.01 to 1.12$)$ & ref & 0.76 (0.66 to 0.88$)$ \\
\hline Carperitide & $2.02(1.91$ to 2.15$)$ & 1.39 (1.33 to 1.45$)$ & ref & 0.35 (0.29 to 0.41$)$ \\
\hline Heparin & $1.73(1.63$ to 1.84$)$ & 1.44 (1.38 to 1.51$)$ & ref & 0.45 (0.39 to 0.52$)$ \\
\hline ISDN or NTG & $1.41(1.32$ to 1.50$)$ & $1.22(1.16$ to 1.28$)$ & ref & 0.53 (0.44 to 0.63$)$ \\
\hline Nicorandil & 1.47 (1.30 to 1.67$)$ & 1.20 (1.08 to 1.34$)$ & ref & $0.20(0.10$ to 0.40$)$ \\
\hline \multicolumn{5}{|l|}{ Inotropes } \\
\hline Dobutamine & 1.49 (1.36 to 1.63$)$ & 1.48 (1.37 to 1.59$)$ & ref & 0.69 (0.52 to 0.90$)$ \\
\hline Dopamine & $0.71(0.65$ to 0.78$)$ & $1.08(1.01$ to 1.15$)$ & ref & 0.79 (0.64 to 0.98$)$ \\
\hline Norepinephrine & 1.41 (1.25 to 1.59$)$ & 1.24 (1.12 to 1.37$)$ & ref & 1.09 (0.80 to 1.48$)$ \\
\hline Milrinone & $0.87(0.72$ to 1.06$)$ & 0.91 (0.79 to 1.05$)$ & ref & $0.36(0.18$ to 0.74$)$ \\
\hline Olprinone & 1.89 (1.43 to 2.50$)$ & $0.82(0.62$ to 1.09$)$ & ref & 0.50 (0.16 to 1.58$)$ \\
\hline Digoxin & $0.85(0.75$ to 0.95$)$ & $1.06(0.98$ to 1.15$)$ & ref & 1.01 (0.79 to 1.29$)$ \\
\hline Calcium channel blockers & 2.21 (1.96 to 2.49$)$ & 1.39 (1.25 to 1.55$)$ & ref & 0.68 (0.46 to 1.02$)$ \\
\hline \multicolumn{5}{|l|}{ Discharge medications } \\
\hline Diuretics & 1.51 (1.37 to 1.66$)$ & 1.14 (1.07 to 1.22$)$ & ref & 0.63 (0.53 to 0.74$)$ \\
\hline ACEls & $1.24(1.16$ to 1.33$)$ & $1.00(0.95$ to 1.06$)$ & ref & $0.44(0.35$ to 0.55$)$ \\
\hline ARBs & 1.16 (1.09 to 1.23$)$ & $1.12(1.06$ to 1.17$)$ & ref & 0.75 (0.65 to 0.87$)$ \\
\hline ACEI or ARBs & 1.35 (1.27 to 1.43$)$ & $1.11(1.06$ to 1.16$)$ & ref & 0.56 (0.48 to 0.64$)$ \\
\hline Aldosterone receptor blockers & 1.30 (1.23 to 1.38$)$ & 1.15 (1.09 to 1.20$)$ & ref & 0.64 (0.55 to 0.74$)$ \\
\hline Digitalis & 0.84 (0.77 to 0.92$)$ & $1.03(0.96$ to 1.10$)$ & ref & $1.23(1.02$ to 1.48$)$ \\
\hline$\beta$-blockers & 1.68 (1.58 to 1.78$)$ & 1.26 (1.20 to 1.32$)$ & ref & 0.49 (0.42 to 0.58$)$ \\
\hline Nitrates & 0.99 (0.91 to 1.07$)$ & 0.97 (0.91 to 1.03$)$ & ref & 1.37 (1.16 to 1.62$)$ \\
\hline Calcium channel blockers & $1.13(1.05$ to 1.21$)$ & $1.14(1.08$ to 1.20$)$ & ref & 0.93 (0.79 to 1.09$)$ \\
\hline Statins & 1.40 (1.31 to 1.50$)$ & 1.23 (1.16 to 1.29$)$ & ref & $0.52(0.42$ to 0.64$)$ \\
\hline Warfarin & 1.35 (1.27 to 1.44$)$ & 1.16 (1.11 to 1.22$)$ & ref & 0.70 (0.59 to 0.82$)$ \\
\hline Antiplatelets & 1.29 (1.22 to 1.38$)$ & $1.12(1.07$ to 1.18$)$ & ref & 0.59 (0.50 to 0.68$)$ \\
\hline Oral inotropic agents & 1.19 (1.06 to 1.34$)$ & $1.13(1.03$ to 1.24$)$ & ref & 0.61 (0.43 to 0.88$)$ \\
\hline
\end{tabular}

The ORs were adjusted for sex and age-group $(<60, \geq 60, \geq 70, \geq 80$, and $\geq 90$ years) using multivariable regression analyses.

ACEIs, ACE inhibitors; ARBs, angiotensin-receptor blockers; CRT-D, cardiac resynchronisation therapy defibrillator; ICD, implantable cardioverter-defibrillator; ISDN, isosorbide dinitrate; NTG, nitrogrycelin; ref, reference.

age-adjusted ORs, was observed to be associated with a higher number of cardiologists. When compared with the 1-4 cardiologists group, hospitals with no cardiologists were less likely to provide these treatments, whereas the 5-9 cardiologists group and the $\geq 10$ cardiologists group were more likely to provide specialty procedures or new drugs, and less likely to provide conventional drugs (eg, intravenous dopamine or digoxin, digitalis at discharge). Furthermore, the outcome measure of patient risk-adjusted mortality also decreased with increasing numbers of cardiologists. These results support those of prior studies where the case volume was shown to be associated with better care processes and outcomes in congestive patients with $\mathrm{HF}^{15}$ and high physician volume, especially with cardiologists, was shown to be associated with lower mortality rates. ${ }^{16}$ However, it should be noted that these results do not unequivocally indicate that a higher number of cardiologists induces higher quality of care. Elderly patients or terminally ill patients are more likely to undergo less invasive treatment, which can be provided in smaller hospitals with fewer cardiologists. Owing to Japan's rapidly ageing population, our results may also be indicative of this treatment style. 
Figure 2 Hospital distribution patterns for specific practices, categorised by the number of cardiologists. (A) Convex pattern (representing commonly used medical therapies). (B) Linear pattern (representing diffusion process of a new therapeutic practice). (C) Concave pattern (representing not widely used medical therapies).
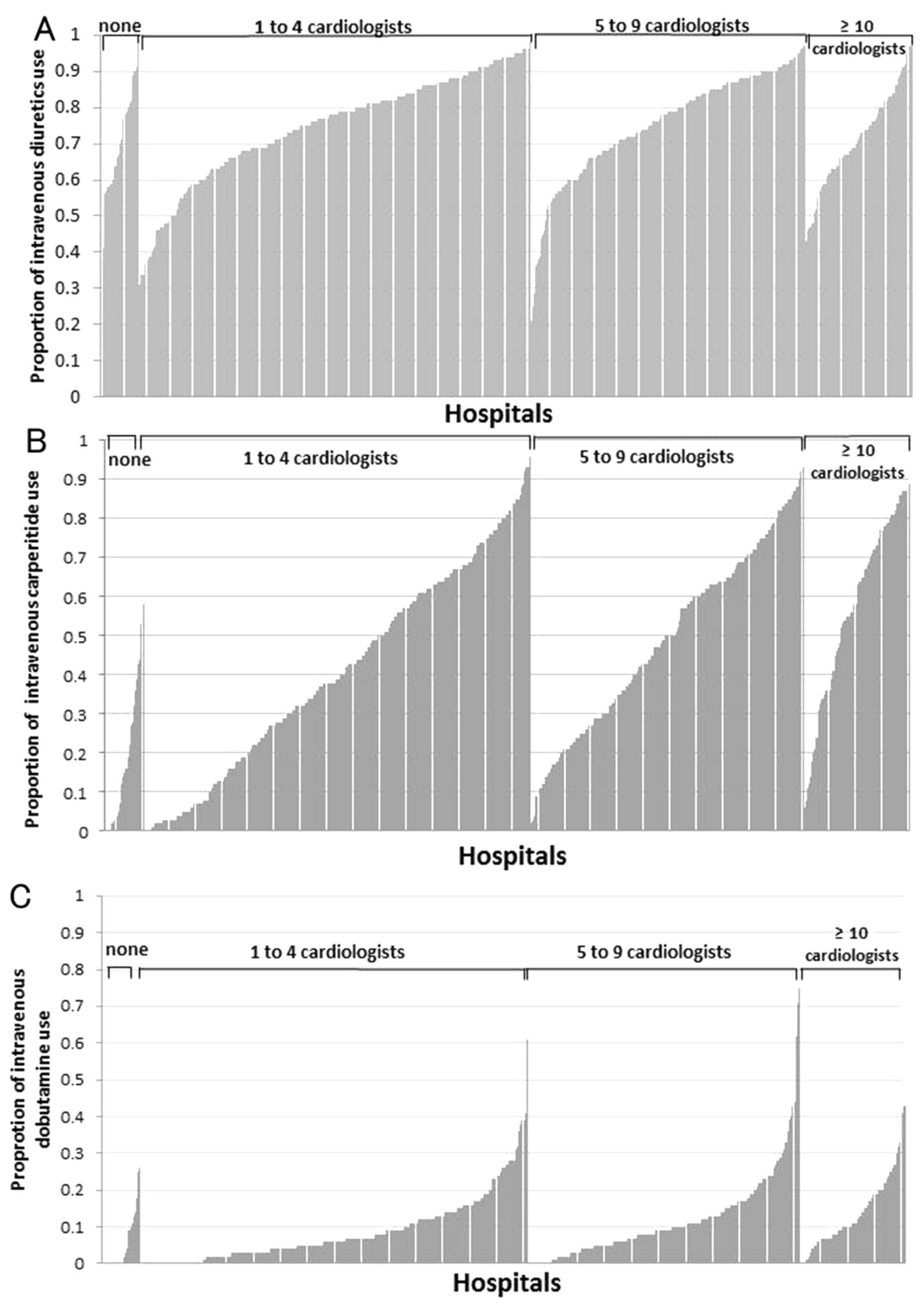

Additionally, our results showed that the lower case volume per cardiologist was related to lower adjusted mortality. The result initially seemed to be contrary to the frequently reported relationship between case volume and outcomes per specialist in major surgeries and cardiovascular interventions. ${ }^{17} 18$ These previous studies have used hospital case volume or case volume per physician as a measure of experience with managing diseases. However, the total number of cardiologists per hospital may be better suited to describe the quality of care in specific diseases that require teams of specialists. Our findings here and in a previous study ${ }^{19}$ are therefore not necessarily contradictory to these prior reports. ${ }^{17} 18$ Moreover, the quality of care shown by the total number of cardiologists may expand the contents of new draft guidelines from the National Institute for Health and Care Excellence (NICE) ${ }^{20}$ in which patients with AHF are recommended to be seen by specialist teams. ${ }^{21}$

The number of cardiologists is very important in medical emergencies such as AHF or AMI which require immediate intervention and the integrated teamwork of cardiovascular specialists and medical staff with $24 \mathrm{~h}$ coverage. The results from our study may lead to the concept of 'resource dependency' as a source of practice variation. This type of care may be considered to be directly affected by the presence and quantity of resources available, and is distinct from individual physicians' skill or experience. Resource dependency can well explain practice variations before supplier-inducement or patient preferences can influence variations. In other words, the availability of manpower resources may affect the quality of care, leading to practice variations among hospitals.

Finally, we found that the three hospital distribution patterns for specific interventions can be used as a tool to capture the diffusion process of a new therapeutic practice. The concept of individual hospital distribution patterns related to the proportion of therapeutic intervention can be illustrated as online supplementary figure S2. Therapies that are not widely used may show 
the concave distribution pattern (type $\mathrm{C}$ ) at first, and would shift from type $\mathrm{C}$ to type $\mathrm{B}$, and finally to type $\mathrm{A}$, when they gradually become more familiar and widespread.

By referring to these three distribution patterns during analyses of cross-sectional data, we may discern how much and how widely a certain therapy is currently adopted among hospitals at a particular time. For example, intravenous carperitide, a recombinant form of atrial natriuretic peptide, which exhibited the intermediate-distribution-stage pattern, has been believed to expand in daily practice in Japan, ${ }^{3}{ }^{4}$ yet the characteristics of hospitals that had used this drug remained unclear. Interestingly, the results from our study revealed that the drug had been much less used among hospitals with fewer cardiologists when compared with the ATTEND registry, which included hospitals with a larger number of cardiologists. In the context of widely known 'innovation diffusion theories, ${ }^{22}{ }^{23}$ this intermediate-distribution-stage pattern may represent a snapshot of the diffusion process of a new therapeutic practice across multiple facilities over time. Furthermore, these results may be utilised to improve currently provided care from the viewpoint of practice guideline adherence or policymaking perspectives.

\section{Study limitations}

There are several limitations to this study. First, hospitals in this study are restricted to some part of those who actively adopt the DPC system. In addition, the clinical circumstances including the use of drugs may differ across the countries. These may limit the generalisability of our results in worldwide clinical settings. Second, when adjusting outcome measures, we did not consider hospital-level factors such as teaching status, urban location, and the presence of a cardiac intensive care unit, which may also have affected the quality of care. Finally, we could not identify the number of cardiologists who were actually treating patients with AHF, differences in competency among individual cardiologists, and the area of cardiovascular subspecialty of each cardiologist. Further studies are required to examine the effect of these issues on quality of care.

\section{CONCLUSIONS}

We revealed wide therapeutic practice variations of AHF in association with the number of cardiologists per facility using an administrative database. Recommended therapeutic practices tended to be provided more frequently in hospitals with more cardiologists. Quality of AHF care may be dependent on manpower resources, and further studies are needed to clarify their relationship.

\footnotetext{
Author affiliations

${ }^{1}$ Department of Healthcare Economics and Quality Management, Kyoto University Graduate School of Medicine, Kyoto, Japan

${ }^{2}$ Department of Health Policy and Informatics, Graduate School of Medical and Dental Sciences, Tokyo Medical and Dental University, Tokyo, Japan
}

${ }^{3}$ Division of Cardiology, National Hospital Organization Osaka National Hospital, Osaka, Japan

${ }^{4}$ Department of Cardiovascular Medicine, Kyoto University Graduate School of Medicine, Kyoto, Japan

Contributors NS and YI had full access to all the data in the study and take responsibility for the analysis and interpretation. NS, YY, YI were involved in the conception and design; NS, HI, KF, YI were involved in the acquisition of data; NS, SK, HI, YI were involved in the analysis and interpretation of data; NS, YI were involved in the drafting of the manuscript; TK and TO critically reviewed the manuscript; NS, SK, HI, YI were involved in the statistical analysis; KF, YI were involved in the obtaining funding. All the authors were involved in critical revisions and approving the final manuscript for publication.

Funding This research was financially supported in part by Health Sciences Research Grants from the Ministry of Health, Labour and Welfare of Japan (H22-iryo-ippan-017, H25-seisaku-shitei-010), and a Grant-in-Aid for Scientific Research from the Japan Society for the Promotion of Science ((A)25253033).

Competing interests None.

Ethics approval This study was approved by the Ethics Committee of Kyoto University Graduate School and Faculty of Medicine, Japan.

Provenance and peer review Not commissioned; externally peer reviewed.

Data sharing statement No additional data are available.

Open Access This is an Open Access article distributed in accordance with the Creative Commons Attribution Non Commercial (CC BY-NC 4.0) license, which permits others to distribute, remix, adapt, build upon this work noncommercially, and license their derivative works on different terms, provided the original work is properly cited and the use is non-commercial. See: http:// creativecommons.org/licenses/by-nc/4.0/

\section{REFERENCES}

1. Lloyd-Jones D, Adams RJ, Brown TM, et al.; Writing Group Members. Heart disease and stroke statistics-2010 update: a report from the American Heart Association. Circulation 2010;121: e46-215.

2. Mebazaa A., ed. Acute heart failure. London: Springer-Verlag Limited, 2008.

3. Sato N, Kajimoto K, Asai K, et al. Acute decompensated heart failure syndromes (ATTEND) registry. A prospective observational multicenter cohort study: rationale, design, and preliminary data. Am Heart J 2010;159:949-55.

4. Sato N, Kajimoto K, Keida T, et al. Clinical features and outcome in hospitalized heart failure in Japan (from the ATTEND registry). Circ $J$ 2013;77:944-51.

5. Tsutsui $\mathrm{H}$, Tsuchihashi-Makaya M, Kinugawa $\mathrm{S}$, et al. Clinical characteristics and outcome of hospitalized patients with heart failure in Japan. Circ J 2006;70:1617-23.

6. Tsuchihashi-Makaya M, Hamaguchi S, Kinugawa S, et al Characteristics and outcomes of hospitalized patients with heart failure and reduced vs preserved ejection fraction. Report from the Japanese Cardiac Registry of Heart Failure in Cardiology (JCARE-CARD). Circ J 2009;73:1893-900.

7. Sasaki N, Lee J, Park S, et al. Development and validation of an acute heart failure-specific mortality predictive model based on administrative data. Can J Cardiol 2013;29:1055-61.

8. Hamada H, Sekimoto M, Imanaka Y. Effects of the per diem prospective payment system with DRG-like grouping system (DPC/ PDPS) on resource usage and healthcare quality in Japan. Health Policy 2012;107:194-201.

9. McKee PA, Castelli WP, McNamara PM, et al. The natural history of congestive heart failure: the Framingham study. $N$ Engl $J$ Med 1971;285:1441-6.

10. Japanese Circulation Society. http://www.j-circ.or.jp/information/ senmoni/kensaku/senmoni_kensaku.htm (accessed 30 Sep 2014). (In Japanese).

11. Ministry of Health, Labour and Welfare. Survey of Medical Institutions. 2012. http://www.e-stat.go.jp/SG1/estat/NewList.do? tid=000001030908 (accessed 30 Sep 2014). (In Japanese).

12. Abraham WT, Fonarow GC, Albert NM, et al. Predictors of in-hospital mortality in patients hospitalized for heart failure: insights from the organized program to initiate lifesaving treatment in hospitalized patients with heart failure (OPTIMIZE-HF). J Am Coll Cardiol 2008;52:347-56. 
13. Nieminen MS, Brutsaert D, Dickstein K, et al. EuroHeart Failure Survey II (EHFS II): a survey on hospitalized acute heart failure patients: description of population. Eur Heart $J$ 2006;27:2725-36.

14. Romano PS, Roos LL, Luft HS, et al. A comparison of administrative versus clinical data: coronary artery bypass surgery as an example. Ischemic Heart Disease Patient Outcomes Research Team. J Clin Epidemiol 1994;47:249-60.

15. Joynt KE, Orav EJ, Jha AK. The association between hospital volume and processes, outcomes, and costs of care for congestive heart failure. Ann Intern Med 2011;154:94-102.

16. Joynt KE, Orav EJ, Jha AK. Physician volume, specialty, and outcomes of care for patients with heart failure. Circ Heart Fail 2013;6:890-7.

17. Hannan EL, Wu C, Ryan TJ, et al. Do hospitals and surgeons with higher coronary artery bypass graft surgery volumes still have lower risk-adjusted mortality rates? Circulation 2003:108:795-801.

18. Hannan EL, Wu C, Walford G, et al. Volume-outcome relationships for percutaneous coronary interventions in the stent era. Circulation 2005;112:1171-9.

19. Park S, Sasaki N, Morishima T, et al. The number of cardiologists, case volume, and in-hospital mortality in acute myocardial infarction patients. Int J Cardiol 2013;168:4470-1.

20. Acute heart failure: diagnosing and managing acute heart failure in adults. http://guidance.nice.org.uk/CG/Wave0/608 (accessed 30 Sep 2014).

21. Wise J. Acute heart failure patients should be seen by specialist teams, says NICE. BMJ 2014;348:93072.

22. Rogers EM. Diffusion of innovations. 5th edn. New York: Free Press, 2003.

23. Berwick DM. Disseminating innovations in health care. JAMA 2003;289:1969-75 